\title{
Unambiguous evidence for magnetoelectric coupling of multiferroic origin in $0.73 \mathrm{BiFeO}_{3}-0.27 \mathrm{PbTiO}_{3}$
}

\author{
Shuvrajyoti Bhattacharjee, ${ }^{1}$ V. Pandey, ${ }^{2}$ R. K. Kotnala, ${ }^{2}$ and Dhananjai Pandey ${ }^{1, a)}$ \\ ${ }^{1}$ School of Materials Science and Technology, Institute of Technology, Banaras Hindu University, \\ Varanasi 221305, India \\ ${ }^{2}$ National Physical Laboratory, K. S. Krishna Marge, Pusa, New Delhi 110012, India
}

(Received 7 October 2008; accepted 17 December 2008; published online 9 January 2009)

\begin{abstract}
Magnetization, frequency dependent dielectric, and structural studies on $0.73 \mathrm{BiFeO}_{3}-0.27 \mathrm{PbTiO}_{3}$ in the temperature range from 300 to $600 \mathrm{~K}$ reveal anomalies in the unit cell parameters and the intrinsic value of the dielectric constant, free from space charge contributions, at the antiferromagnetic transition temperature $\left(T_{N}\right)$. Our results provide unambiguous confirmation of magnetoelectric coupling of multiferroic origin at $T_{N}$ and evidence for monoclinic distortion of the ferroelectric phase. (C) 2009 American Institute of Physics. [DOI: 10.1063/1.3068000]
\end{abstract}

Bismuth based transition metal oxides $\left(\mathrm{BiBO}_{3}\right)$ and their solid solutions with other perovskites are known for their important multiferroic properties (see the recent reviews) $)^{1-3}$ where the ferroelectricity arises due to the $6 s$ lone pair chemistry of $\mathrm{Bi}^{+3}$, while the magnetic order results from the $\mathrm{Fe} /$ $\mathrm{Mn} / \mathrm{Cr}$ ions occupying the $B$ site. Of these compounds, $\mathrm{BiFeO}_{3}$ is of special interest as its ferroelectric and magnetic transition temperatures are located well above the room temperature, raising possibilities of room temperature multiferroic devices. ${ }^{1-3}$ Bulk $\mathrm{BiFeO}_{3}$ shows a rhombohedrally distorted perovskite structure in the $R 3 c$ space group with a ferroelectric transition temperature $T_{C}$ of $\sim 1103 \mathrm{~K}$ (Ref. 4) and $G$-type antiferromagnetic (AFM) spin configuration with an incommensurate cycloidal spin structure ${ }^{5}$ with a magnetic $T_{N} \sim 643 \mathrm{~K}^{6}{ }^{6}$ The long period modulated magnetic structure leads to the cancellation of net macroscopic magnetization and hence the absence of linear magnetoelectric coupling. ${ }^{1}$ The linear magnetoelectric effect has been reported in bulk $\mathrm{BiFeO}_{3}$ after breaking the modulated magnetic structure in the presence of high external magnetic field. ${ }^{7,8}$ It has been suggested that chemical substitutions may also suppress the cycloidal spin structure. ${ }^{9-11}$

The observation of an anomaly in the temperature dependence of dielectric constant around the magnetic transition temperature is often taken as an evidence for magnetoelectric coupling. ${ }^{12,13}$ However, a dielectric anomaly at the magnetic transition temperature can also result from the magnetoresistance effects in granular systems ${ }^{14}$ because of the large difference in the resistivities of the grains and grain boundaries. For an unambiguous confirmation of multiferroic coupling, it is therefore essential to demonstrate that the observed dielectric anomaly at the magnetic transition temperature is not due to the magnetoresistance at various interfaces such as grain boundaries. It should also be supported by other auxiliary evidence such as magnetoelastic coupling in the bulk phase through structural studies. ${ }^{11}$

Here, we present the results of magnetization, dielectric, and structural studies as a function of temperature on $0.73 \mathrm{BiFeO}_{3}-0.27 \mathrm{PbTiO}_{3}$ (BF-0.27PT) ceramics, which reveal monoclinic distortion of the ferroelectric phase and in-

\footnotetext{
a) Author to whom correspondence should be addressed. Electronic mail: dpandey_bhu@yahoo.co.in.
}

trinsic coupling between the AFM and ferroelectric order parameters. The particular composition $(x=0.27)$ that we have investigated is of special interest as it corresponds to the highest value of $x$ closest to the morphotropic phase boundary (MPB) in the BF- $x$ PT system ${ }^{15,16}$ before the structure transforms to the tetragonal phase. The proximity of MPB is expected to influence multiferroic coupling effects as well. There is no report of intrinsic magnetoelectric coupling in the BF- $x$ PT system for a single phase composition (i.e., $x$ $=0.27$ ) closest to the MPB. The previous dielectric and magnetic studies on this system were not conclusive since the samples used contained coexisting tetragonal $(T)$ and rhombohedral $(R)$ phases over a very wide composition range $(0.2 \leq x \leq 0.4){ }^{10,17}$ whereas the intrinsic width of the MPB region is $\sim 3 \%$ only. ${ }^{15}$

Monophasic samples of BF-0.27PT prepared by solid state route ${ }^{18}$ were used in this investigation. The density of the sintered pellets was $\sim 98 \%$ of the theoretical density. The high temperature $\mathrm{x}$-ray diffraction (XRD) data were collected using an $18 \mathrm{~kW}$ Cu-rotating anode based Rigaku (Tokyo, Japan) powder diffractometer with a high temperature attachment in the temperature range from 300 to $875 \mathrm{~K}$ and a $2 \theta$ range of $15^{\circ}-120^{\circ}$ at a scan step of $0.02^{\circ}$. The XRD data were analyzed by Rietveld and Le Bail refinement techniques using FULLPROF package. ${ }^{19}$ Dielectric and impedance measurements were carried out using a Nova Control (Alpha-A) high performance frequency analyzer in the temperature range from 300 to $673 \mathrm{~K}$ at a heating rate of 1 $\mathrm{K} / \mathrm{min}$. The $M-H$ loop at room temperature and temperature dependent magnetization measurements in the temperature range from 300 to $673 \mathrm{~K}$ at a magnetic field of 2500 Oe were carried out using vibrating sample magnetometer (VSM7305 , Lakeshore) at a heating rate of $5 \mathrm{~K} / \mathrm{min}$.

The temperature variation of magnetic susceptibility $(\chi)$ of BF-0.27PT depicted in Fig. 1(a) shows an anomaly at around $\sim 473 \mathrm{~K}$ due to an AFM transition, as confirmed by the observation of a double hysteresis loop at room temperature [see the inset in Fig. 1(a)]. To demonstrate the coupling between ferroelectric and AFM order parameters, we depict in Figs. 1(b) and 1(c) the temperature dependence of the real $\left(\varepsilon^{\prime}\right)$ and imaginary $\left(\varepsilon^{\prime \prime}\right)$ parts of the dielectric constant across the AFM transition temperature $T_{N}$. Both $\varepsilon^{\prime}$ and $\varepsilon^{\prime \prime}$ show anomalies in the vicinity of $T_{N}$ similar to that observed in 


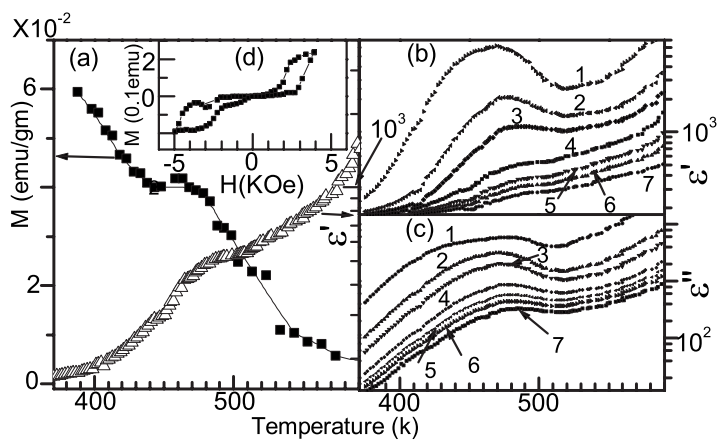

FIG. 1. Temperature dependence of the (a) magnetization $(M)$ and (b) real $\left(\varepsilon^{\prime}\right)$ and (c) imaginary $\left(\varepsilon^{\prime \prime}\right)$ parts of the dielectric constant. The inset (d) shows AFM hysteresis loop at room temperature. The variation in $\varepsilon^{\prime}$ across $T_{N}$ at $300 \mathrm{kHz}$ is also shown in (a). The $1-7 \varepsilon^{\prime}$ and $\varepsilon^{\prime \prime}$ semilogarithmic plots correspond to $10,50,100,300,500,700$, and $1000 \mathrm{kHz}$, respectively.

other multiferroics. However, the peak value of $\varepsilon^{\prime}$ at $T_{N}$ over and above the rising background value in our case is very large $(\sim 4400)$ at $100 \mathrm{~Hz}$ and it decreases to $\sim 60$ at $300 \mathrm{kHz}$. We now proceed to discuss the origin of this huge frequency dependent dielectric anomaly at $T_{N}$.

The high value of permittivity at low frequencies may not be entirely of multiferroic origin. The Cole-Cole complex impedance plots shown in Fig. 2 at two different temperatures, $473 \mathrm{~K}$ (i.e., at $T_{N}$ ) and $481 \mathrm{~K}$ (above $T_{N}$ ), distinctly show three separate semicircular arcs characteristic of electrode-grain interface (circle 1), grain boundary (circle 2), and grain contributions (circle 3) to the total polarization in the increasing order of frequency. ${ }^{20}$ The observation of three distinct polarization processes is linked with the loss of oxygen during firing at high temperatures, ${ }^{21,11}$ which can be written as

$$
\mathrm{O}_{\mathrm{O}}=\frac{1}{2} \mathrm{O}_{2}+V_{\mathrm{O}}^{\circ}+2 e^{\prime}
$$

in the Kröger-Vink notation. The electrons released in the above reaction may be captured by $\mathrm{Fe}^{3+}$ leading to the formation of $\mathrm{Fe}^{2+}$. This leads to hopping of electrons among the two different valence states, which increases conductivity of the grains. During cooling down period after sintering, the reverse reaction occurs. However due to insufficient time available during cooling, the reoxidation occurs preferentially at grain boundaries only. Thus grain boundaries regain

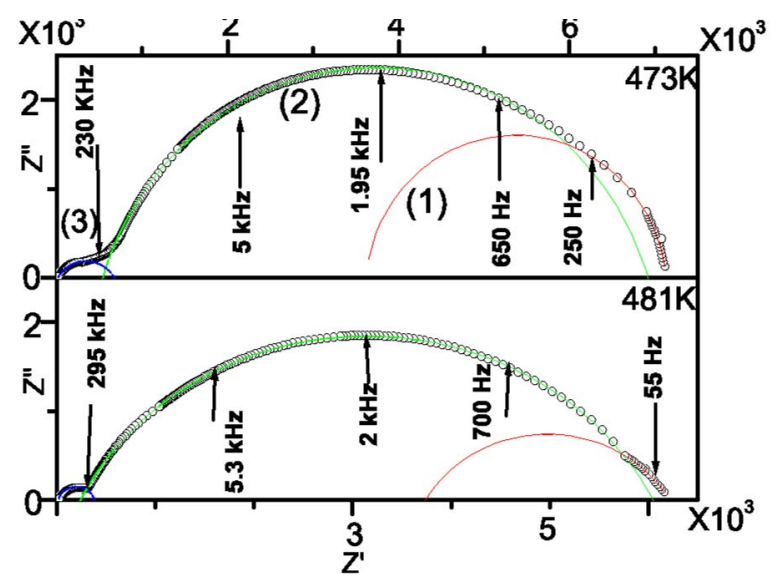

FIG. 2. (Color online) Complex impedance plots $\left(Z^{\prime \prime}\right.$ vs $\left.Z^{\prime}\right)$ at (a) 473 and (b) $481 \mathrm{~K}$. Symbols are experimental data points, while the semicircular arcs are the fitted curves.

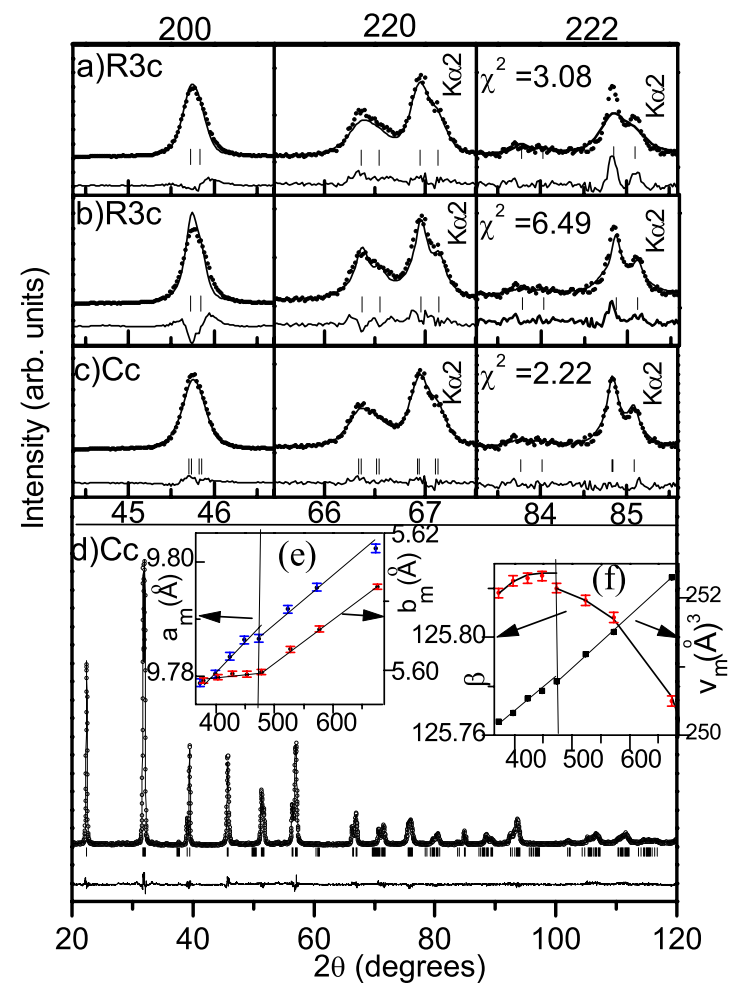

FIG. 3. (Color online) Observed (dots), calculated (line), and difference (bottom curve) profiles for the pseudocubic 200, 220, and 222 peaks obtained after Rietveld refinement of powder diffraction data in the $2 \theta$ range from 20 to $120^{\circ}$ for rhombohedral $R 3 c$ space group (a) with anisotropic and (b) without anisotropic peak broadening function and (c) using monoclinic $C c$ space group. The overall Rietveld fit for the $C c$ space group is shown in (d). The insets in (d) show the temperature dependence of (e) the monoclinic $a_{m}$ and $b_{m}$ parameters and (f) the unit cell volume and monoclinic distortion angle $\beta$.

their insulating character, while the grains remain more or less semiconducting. The grain boundaries thus act as barriers against the hopping electrons, which pile up against them leading to space charge polarization. A similar space charge polarization occurs at the electrode-grain interface. These space charge polarization processes are responsible for the extremely large value of dielectric constant at very low and medium frequencies. ${ }^{20}$ However, at high frequencies (responsible for the smallest circle in Fig. 2), the space charge polarizations are not able to contribute. Thus the dielectric anomaly observed at $T_{N}$ for $300 \mathrm{kHz}$ [shown separately in Fig. 1(a)] is free from the extrinsic (space charge) contributions. The dielectric anomaly due to the intrinsic multiferroic coupling is quite prominent in the sense that the change $\left(\Delta \varepsilon^{\prime}\right)$ in $\varepsilon^{\prime}$ at $T_{N}$ with respect to the rising background is $\sim 60$ at $300 \mathrm{kHz}$ [see Fig. 1(a)], as compared to other multiferroic materials where it is much less pronounced [e.g., $\sim 14$ at $75 \mathrm{kHz}$ for $\mathrm{YMnO}_{3}$ (Ref. 13)].

The anomaly in $\varepsilon^{\prime}$ due to the intrinsic grain contributions at $T_{N}$ corresponds to the additional polarization of the lattice. Such a polarization is expected to lead to the distortion of the lattice and hence anomalies in the unit cell parameters also. ${ }^{11}$ To capture such anomalies, we have analyzed the powder diffraction data at various temperatures using Rietveld and Le Bail refinement techniques. Since the space group of the room temperature ferroelectric phase of $\mathrm{BiFeO}_{3}$ is believed to be $R 3 c,{ }^{5}$ we first considered this space group in our refinements. Figure 3(a) depicts the observed, 
TABLE I. Refined structural parameters of $\mathrm{BiFeO}_{3}-0.27 \mathrm{PbTiO}_{3}$ using $C c$ space group.

\begin{tabular}{|c|c|c|c|c|}
\hline \multirow{2}{*}{$\begin{array}{l}a_{m}=9.7781(6) \\
\alpha=\gamma=90 \\
\text { Ions }\end{array}$} & $\AA ̊ 丿, b_{m}=5.5888(3)$ & \multicolumn{3}{|c|}{$\AA, c_{m}=5.6311(2) \AA ⿻$, $\beta=125.793(3), \operatorname{deg}$} \\
\hline & $x$ & $y$ & $z$ & $B\left(\AA^{2}\right)$ \\
\hline $\mathrm{Ti}^{+4} / \mathrm{Fe}^{+3}$ & $0.272(2)$ & $0.254(4)$ & $0.726(4)$ & $0.12(8)$ \\
\hline $\mathrm{Bi}^{+3} / \mathrm{Pb}^{+2}$ & 0.000 & 0.25000 & 0.000 & $1.50(2)$ \\
\hline $\mathrm{O}_{\mathrm{I}}^{-2}$ & $-0.016(5)$ & $0.25(1)$ & $0.57(6)$ & $0.2(7)$ \\
\hline $\mathrm{O}_{\mathrm{II}}^{-2}$ & $0.337(4)$ & $0.449(7)$ & $0.060(7)$ & $0.2(8)$ \\
\hline $\mathrm{O}_{\mathrm{III}}^{-2}$ & $0.301(7)$ & $0.04(1)$ & $-0.04(1)$ & $1.5(1.3)$ \\
\hline & $R_{p}=10.5, R_{\mathrm{wp}}=$ & $R_{\text {expt }}=8.7$, and & $\chi^{2}=2.22$ & \\
\hline
\end{tabular}

calculated, and difference profiles, obtained by full pattern Rietveld refinement, of a few selected reflections $(200,220$, and 222), for the $R 3 c$ space group. For the $R 3 c$ space group, the 200 peak is expected to be singlet, whereas 220 and 222 should be doublets, as is apparently the case in the experimental data shown in Fig. 3. However, we find that the h00-type and hh0-type peaks (e.g., 200 and 220) show anomalous broadening. For example, the full width at half maximum of 200 peak is far greater than that of the 222 peak, whereas as per the relationship of Cagliotti et al., ${ }^{22}$ the 222 peak should have been broader. To account for the large anomalous broadening of $h 00$-type and $h h 0$-type peaks, we used the anisotropic strain model of Stephens ${ }^{23}$ in our refinements. It is evident from this figure that although the anisotropic peak broadening of the 200- and 220-type profiles is accounted for, the 222 reflection shows huge mismatch between the observed and calculated profiles. If we do not use the anisotropic peak broadening functions in our refinements, the 222 peak is reasonably accounted for, but mismatch appears for all other peaks [see Fig. 3(b)], leading to a very high $\chi^{2}=6.49$ value, as compared to that $\left(\chi^{2}=3.08\right)$ shown in Fig. 3(a). The monoclinic $C c$ space group, on the other hand, gives excellent fit for all the peaks with a lower $\chi^{2}$ value of 2.22 [see Fig. 3(c)]. As discussed in detail elsewhere, ${ }^{18}$ we obtained nine possible space groups for the ferroelectric phase of BF-0.27PT based on group theoretical considerations using ISOTROPY package, ${ }^{24}$ out of which the $C c$ space group gives the best fit. Figure 3(d) depicts the quality of overall Rietveld fit using the $C c$ space group. Table I lists the refined structural parameters at room temperature. We have also verified that the $C c$ space group for BF-0.27PT does not change above $T_{N}$. The temperature variation of the monoclinic cell parameters, obtained by full profile Le Bail refinements at various temperatures, is depicted in the two insets of Fig. 3(d). The anomalies in the unit cell parameters $\left(a_{m}\right.$, $b_{m}$, and $\left.\beta\right)$ and the unit cell volume $\left(V_{m}\right)$ are clearly seen at
$T_{N}$, confirming the distortion of the lattice accompanying the dielectric anomaly due to the intrinsic multiferroic coupling via magnetoelastic effect. ${ }^{11}$

To summarize, our studies on the BF-0.27PT system reveal anomalies in the intrinsic value of the dielectric constant, free from any space charge contributions, and also the unit cell parameters at the AFM transition temperature $\left(T_{N}\right)$ as a result of multiferroic coupling. We have also shown that the so-called rhombohedral phase of BF- $x$ PT (Refs. 10 and $16)$ is in fact monoclinic in the $C c$ space group. Our results provide unambiguous confirmation of the intrinsic coupling between the ferroelectric and AFM order characteristics of a multiferroic system.

One of the authors (Shuvrajyoti Bhattacharjee) acknowledges the award from Senior Research Fellowship of CSIR, India.

${ }^{1}$ W. Eerenstein, N. D. Mathur, and J. F. Scott, Nature (London) 442, 759 (2006).

${ }^{2}$ S. W. Cheong and M. Mostovoy, Nature Mater. 6, 13 (2007).

${ }^{3}$ R. Ramesh and N. A. Spaldin, Nature Mater. 6, 21 (2007).

${ }^{4}$ Yu. E. Roginskaya, Yu. Ya. Tomashpolskii, Yu. N. Venevtsev, V. M. Petrov, and G. S. Zhdanov, Sov. Phys. JETP 23, 47 (1966).

${ }^{5}$ I. Sosnowska, T. P. Neumair, and E. Steichele, J. Phys. C 15, 4835 (1982).

${ }^{6}$ W. Kaczmarek, Z. Pajak, and M. Polomska, Solid State Commun. 17, 807 (1975).

${ }^{7}$ Yu. F. Popov, A. K. Zvezdin, G. P. Vorob'ev, A. M. Kadomtseva, V. A. Murashev, and D. N. Rakov, JETP Lett. 57, 69 (1993).

${ }^{8}$ B. Ruette, S. Zvyagin, A. P. Pyatakov, A. Bush, J. F. Li, V. I. Belotelov, A. K. Zvezdin, and D. Viehland, Phys. Rev. B 69, 064114 (2004).

${ }^{9}$ N. Wang, J. Cheng, A. Pyatakov, A. K. Zvezdin, J. F. Li, L. E. Cross, and D. Viehland, Phys. Rev. B 72, 104434 (2005).

${ }^{10}$ W. M. Zhu, H. Y. Guo, and Z. G. Ye, Phys. Rev. B 78, 014401 (2008).

${ }^{11}$ A. Singh, V. Pandey, R. K. Kotnala, D. Pandey, Phys. Rev. Lett. 101, 247602 (2008).

${ }^{12}$ D. L. Fox, D. R. Tilley, J. F. Scott, and H. J. Guggenheim, Phys. Rev. B 21, 2926 (1980).

${ }^{13}$ Z. J. Huang, Y. Cao, Y. Y. Sun, Y. Y. Xue, and C. W. Chu, Phys. Rev. B 56, 2623 (1997).

${ }^{14}$ G. Catalan, Appl. Phys. Lett. 88, 102902 (2006).

${ }^{15}$ S. Bhattacharjee, S. Tripathi, and D. Pandey, Appl. Phys. Lett. 91, 042903 (2007).

${ }^{16}$ R. T. Smith, G. D. Achenbach, R. Gerson, and W. J. James, J. Appl. Phys. 39, 70 (1968).

${ }^{17}$ W. M. Zhu and Z. G. Ye, Ceram. Int. 30, 1435 (2004).

${ }^{18} \mathrm{~S}$. Bhattacharjee and D. Pandey (unpublished).

${ }^{19}$ J. Rodriguez-Carvajal Laboratory, FULLPROF, a Rietveld and pattern matching analysis program, Laboratoire Leon Brillouin, CEA-CNRS, France.

${ }^{20}$ A. K. Joncher, Dielectric Relaxationin Solids (Chelser Dielectric, London, 1983).

${ }^{21}$ S. P. Singh, A. K. Singh, D. Pandey, H. Sharma, and O. Prakas, J. Mater. Res. 18, 2677 (2003).

${ }^{22}$ G. Caglioti, A. Paoletti, and F. P. Ricci, Nucl. Instrum. 3, 223 (1958).

${ }^{23}$ P. W. Stephens, J. Appl. Crystallogr. 32, 281 (1999).

${ }^{24}$ H. T. Stokes, D. M. Hatch, and B. J. Campbell, ISOTROPY, 2007 (http:// stokes.byu.edu/isotropy.html). 